\title{
A NOTE ON THE RADICAL OF ROW-FINITE MATRICES
}

\section{by REBECCA SLOVER}

(Received 11 May, 1970)

Let $R$ be an associative ring, $J$ an infinite index set, and $R_{J}$ the ring of all $J \times J$ row-finite matrices over $R$. The Jacobson radical of $R$ will be denoted by $\Gamma(R)$.

In [5] a diagonalized matrix is defined as follows:

Definitron. A row-finite matrix $A$ over $R$ is diagonalized provided that, if $\left\{a_{i_{1} j_{1}}, a_{i_{2} j_{2}}\right.$, $\left.a_{i_{3} j_{3}}, \ldots\right\}$ is a sequence of entries of $A$ such that $\left\{j_{1}, j_{2}, j_{3}, \ldots\right\}$ contains infinitely many distinct elements, then there exists a positive integer $p$ such that $a_{i_{1} j_{1}} a_{i_{2} j_{2}} \ldots a_{i_{p} j_{p}}=0$.

It is shown in [5] that, if $R$ is a commutative ring and $A$ is a row-finite matrix over $R$, then $A$ is in $\Gamma\left(R_{J}\right)$ if and only if $A$ is in $[\Gamma(R)]_{J}$ and $A$ is diagonalized. Utilizing the recent results of N. E. Sexauer and J. E. Warnock, we can now show that it is not necessary to assume that $R$ is commutative.

THEOREM. Let $A$ be an element of $R_{J}$. Then $A$ is in $\Gamma\left(R_{J}\right)$ if and only if $A$ is in $[\Gamma(R)]_{J}$ and each element of the left ideal of $R_{J}$ generated by $A$ is diagonalized.

Proof. Suppose that $A \in \Gamma\left(R_{J}\right)$. E. M. Patterson has shown in [2] that $\Gamma\left(R_{J}\right) \subseteq[\Gamma(R)]_{J}$. Let $B$ be an element of the left ideal of $R_{J}$ generated by $A$. Let $\left\{b_{i_{1} j_{1}}, b_{i_{2} j_{2}}, b_{i_{3} j_{3}}, \ldots\right\}$ be a set of entries of $B$ such that $\left\{j_{1}, j_{2}, j_{3}, \ldots\right\}$ contains infinitely many distinct elements. Let $k(1)=1$. Suppose that $t$ is an integer greater than 1 and that $k(s)$ has been defined for each positive integer $s$ less than $t$. Let $k(t)$ be a positive integer such that $j_{k(t)} \neq j_{k(s)}$ for each positive integer $s$ less than $t$. For each positive integer $s$,

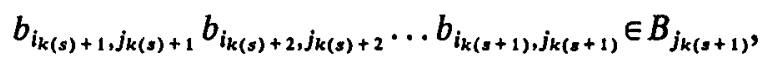

the left ideal of $R$ generated by the elements of the $j_{k(s+1)}$ th column of $B$. Since $B \in \Gamma\left(R_{J}\right)$, by the main theorem of [4] there exists a positive integer $p$ such that $b_{i_{1} j_{1}} b_{i_{2} j_{2}} \ldots b_{i_{p} j_{p}}=0$.

Conversely, suppose that $A$ is an element of $[\Gamma(R)]_{J}$ that is not contained in $\Gamma\left(R_{J}\right)$. By [4, Main Theorem and Proposition 3], there exists a sequence $\left\{b_{i_{1} j_{1}}, b_{i_{2} j_{2}}, b_{i_{3} j_{3}}, \ldots\right\}$ such that, for each positive integer $k, b_{i_{k} j_{k}}=\sum_{h=1}^{s_{k}} x_{h k} a_{h^{\prime} j_{k}}$, where $s_{k}$ is a positive integer, each $x_{h k} \in R$, each $h^{\prime}$ is a positive integer which depends upon $h$, each $a_{h^{\prime} j_{k}}$ is in the $j_{k}$ th column of $A, j_{k} \neq j_{m}$ if $k \neq m$, and $b_{i_{1} j_{1}} b_{i_{2} j_{2}} \ldots b_{i_{n} j_{n}} \neq 0$ for each positive integer $n$. Thus

$$
\sum_{h=1}^{s_{1}} x_{h 1} a_{h^{\prime} j_{1}} b_{i_{2} j_{2}} \ldots b_{i_{n} j_{n}}=b_{i_{1} j_{1}} b_{i_{2} j_{2}} \ldots b_{i_{n} j_{n}} \neq 0
$$

for each positive integer $n$. Since there are only finitely many integers $h$ such that $1 \leqq h \leqq s_{1}$, there exists a positive integer $h_{1} \leqq s_{1}$ such that $x_{h_{1} 1} a_{h_{1}{ }^{\prime} j_{1}} b_{i_{2} j_{2}} \ldots b_{i_{n} j_{n}} \neq 0$ for infinitely many integers $n$ greater than 1. Suppose that, for some positive integer $k$, there exist integers $h_{1}, h_{2}, \ldots, h_{k}$ such that $1 \leqq h_{i} \leqq s_{i}$ for $1 \leqq i \leqq k$ and $x_{h_{1} 1} a_{h_{1}{ }^{\prime} j_{1}} \ldots x_{h_{k} k} a_{h_{k} j_{k}} b_{i_{k+1} j_{k+1}} \ldots b_{i_{n} J_{n}} \neq 0$ for infinitely many integers $n$ greater than $k$. If $h$ is a positive integer not greater than $s_{k+1}$ 
and $n$ is an integer greater than $k$, let $f_{h n}=x_{h_{1} 1} a_{h_{1}^{\prime} j_{1}} \ldots x_{h_{k} k} a_{h^{\prime} k_{k}} x_{h_{1, k+1}} a_{h^{\prime} j_{k+1}} b_{i_{k+2} j_{k+2}} \ldots b_{l_{n} j_{n}}$. Then $\sum_{h=1}^{s_{k+1}} f_{h n}=x_{h_{1} 1} a_{h_{1}^{\prime} j_{1}} \ldots x_{h_{k} k} a_{h_{k^{\prime}} j_{k}} b_{i_{k+1} j_{k+1}} b_{i_{k+2} j_{k+2}} \ldots b_{i_{n} j_{n}} \neq 0$ for infinitely many integers $n$ greater than $k$. Thus there exists a positive integer $h_{k+1}$, not greater than $s_{k+1}$, such that $f_{h_{k+1} n} \neq 0$ for infinitely many integers $n$ greater than $k+1$. Therefore there exists a sequence $\left\{x_{h_{1} 1} a_{h_{1}^{\prime} j_{1}}, x_{h_{22}} a_{h^{\prime} j_{2}}, \ldots\right\}$ such that $x_{h_{1} 1} a_{h_{1} j_{1}} x_{h_{2} 2} a_{h_{2}^{\prime} j_{2}} \ldots x_{h_{n} n} a_{h_{n}^{\prime} j_{n}} \neq 0$ for each positive integer $n$. Well-order $J$. Let $Y=\left(y_{i j}\right)$ be the element of $R_{J}$ defined in the following way. For each positive integer $m$, if $k_{m}$ is the $m$ th element of $J$, let $y_{k_{m} h_{m^{\prime}}}=x_{h_{m} m^{\prime}}$. Let $y_{i j}=0$ for all other members of $J \times J$. Let $Z=Y A$. Then $Z$ is an element in the left ideal of $R_{J}$ generated by $A$ and, if $m$ is a positive integer and $k_{m}$ is the $m$ th element of $J$, then $x_{h_{m} m} a_{h_{m^{\prime}} J_{m}}$ is the $\left(k_{m}, j_{m}\right)$ th entry of $Z$. Since $x_{h_{1} 1} a_{h_{1} j_{1}^{\prime} j_{1}} x_{h_{2} 2} a_{h_{2} j_{2}} \ldots x_{h_{n} n} a_{h^{\prime} j_{n}} \neq 0$ for each positive integer $n$, $Z$ is not diagonalized.

\section{REFERENCES}

1. N. Jacobson, Structure of rings, Amer. Math. Soc. Colloquium Publications 37 (Providence, R.I., 1956).

2. E. M. Patterson, On the radical of certain rings of infinite matrices, Proc. Roy. Soc. Edinburgh Sect. A 65 (1960), 263-271.

3. E. M. Patterson, On the radical of rings of row-finite matrices, Proc. Roy. Soc. Edinburgh Sect. A $66(1961-62), 42-46$.

4. N. E. Sexauer and J. E. Warnock, The radical of row-finite matrices over an arbitrary ring, Trans. Amer. Math. Soc. 139 (1969), 287-295.

5. R. Slover, The Jacobson radical of row-finite matrices, J. of Algebra 12 (1969), 345-359.

Virginia Polytechnic Institute and State University

BLACKSBURG, VIRGINIA 24061 